\title{
Emergencia alimentaria y derecho humano a la alimentación. Un análisis del Programa Argentina contra el Hambre
}

Food emergency and the human right to food. An analysis of the Argentina Hunger Programme

Emergência alimentaria e direito bumano à alimentação. Uma análise do Programa Argentina Contra a Fome

Crise alimentaire et droit bumain à

l'alimentation. Une analyse du programme Argentine contre la faim

粮食紧急情况和食物权 阿根廷反饥饿计划分析

\author{
Ana María Bonet de Viola ${ }^{1}$ \\ Universidad Católica de Santa Fe - Argentina \\ María Eugenia Marichal \\ Universidad Nacional del Litoral - Argentina
}

Revista Derechos en Acción ISSN 2525-1678/ e-ISSN 2525-1686

Año 5/Nº 14, Verano 2019-2020 (21 diciembre a 20 marzo), 480-512

DOl: https://doi.org/10.24215/25251678e366

ORCID: https://orcid.org/0000-0002-9991-5475

https://orcid.org/0000-0002-4250-9337

Recibido: 23/12/2019

Aprobado: 09/02/2020

\footnotetext{
Abogada (Universidad Nacional del Litoral); Doctora en derecho (Dr. jur.), Universidad de Bremen (Alemania) especializada en derechos sociales, particularmente en el derecho a la alimentación. Trabajó sobre todo sobre la colisión entre derechos humanos y economía. En su tesis doctoral abordó la colisión entre el derecho a la alimentación y los sistemas vigentes de propiedad intelectual. (abonet@ucsf.edu.ar)

2 Abogada (Universidad Nacional del Litoral), participa del Centro de Investigación de la Facultad de Ciencias Jurídicas y Sociales de la UNL. (marichal@fcjs.unl.edu.ar)
} 
Resumen: Este trabajo se propone analizar los mecanismos de articulación entre el tratamiento de la emergencia alimentaria y el derecho humano a la alimentación que presenta el PACH en el contexto del ordenamiento jurídico argentino. La concentración de la política alimentaria nacional en programas de emergencia alimentaria significa una limitación del derecho humano a la alimentación a un aspecto básico (derecho a no morir de hambre) el cual, si bien es prioritario, no aborda la cuestión alimentaria en profundidad al deslindarla de otros aspectos sistémicos como son la producción, distribución y consumo de alimentos. La complejidad que amerita el enfoque de la soberanía alimentaria propuesto en el PACH exige la articulación de ese aspecto básico con aquellos aspectos sistémicos. Ello demanda medidas integrales y a largo plazo que aborden la problemática alimentaria desde la integralidad de sus componentes como el acceso a los recursos escenciales para la alimentación.

Palabras clave: Emergencia Alimentaria, Derecho Humano a la Alimentación, Plan Argentina Contra el Hambre.

Abstract: This paper aims to analyze the mechanisms of articulation between the treatment of food emergencies and the human right to food presented by the PACH in the context of the Argentine legal system. The concentration of national food policy on emergency food programs means limiting the human right to food to a basic aspect (the right not to die of hunger) which, although a priority, does not address the food issue in depth by separating it from other systemic aspects such as the production, distribution and consumption of food. The complexity of the approach to food sovereignty proposed in the PACH requires the articulation of this basic aspect with those systemic aspects. This demands comprehensive and long-term measures that address the food problem from the integral nature of its components, such as access to essential food resources.

Keywords: Food Emergency, Human Right to Food, Argentine Plan Against Hunger.

Resumo: Este trabalho tem como objetivo analisar os mecanismos de articulação entre 0 tratamento da emergência alimentaria e 0 direito humano à alimentação que apresenta o Plano Argentina Contra a Fome no contexto do sistema jurídico argentino. A concentração da política 
nacional de alimentos em programas de emergência alimentaria significa uma limitação do direito humano à alimentação a um aspecto básico (direito de não morrer de fome) que, embora seja uma prioridade, não aborda a questão alimentaria profundamente ao separá-la de outros aspectos sistêmicos como são a produção, distribuição e consumo de alimentos. A complexidade que merece a abordagem de soberania alimentaria proposta no Plano requer a articulação desse aspecto básico com esses aspectos sistêmicos. Isso exige medidas integrais e a longo prazo que abordem o problemática alimentaria a partir da integralidade de seus componentes, como 0 acesso a recursos essenciais para a alimentação.

Palavras-chave: Emergência Alimentaria, Direito Humano à Alimentação, Plano Argentina Contra a Fome

Résumé: Ce travail vise à analyser les mécanismes d'articulation entre le traitement de la crise alimentaire et le droit humain à l'alimentation que le "PACH" (Programme Argentine Contre la Faim) présente dans le contexte du système juridique argentin. La concentration de la politique alimentaire nationale dans des programmes d'urgence alimentaire implique une limitation du droit humain à l'alimentation dans un de ses aspects fondamentales (droit de ne pas mourir de faim). Cela, bien que prioritaire, n'aborde pas le problème alimentaire en profondeur en le délimitant d'autres aspects systémiques comme la production, la distribution et la consommation alimentaire. La complexité qui mérite I'approche de la souveraineté alimentaire proposée dans le PACH, exige de l'articulation de cet aspect fondamental avec ces autres aspects systémiques. Cela nécessite des mesures globales et à long terme, qui peuvent aborder le problème alimentaire en tenant compte la totalité de ses composants, comme l'accès aux ressources essentielles pour la nourriture.

Mots clés: Crise alimentaire, droit humain à I'alimentation, plan Argentine contre la faim.

摘要：这项工作旨在分析在阿根廷法律制度的背景下，食品紧急情 况处理所提出的粮食紧急情况处理与食物权之间的联系机制。国家 粮食政策集中在粮食紧急计划中, 意味着将食物权限制在一个基本 方面 (不挨饿的权利), 尽管这是优先事项, 但并未通过划定界限来深 入解决食物问题其他系统性方面, 例如食品的生产, 分配和消费。提 
议的实现食品主权方法的复杂性要求将该基本方面与那些系统方 面相结合。这就要求采取全面和长期的措施，从其组成部分的完整 性来解决粮食问题, 例如获得粮食必需资源。

关键词: 粮食紧急情况, 食物权, 阿根廷反饥饿计划。

\section{Introducción}

La cuestión del acceso a los alimentos constituye una preocupación de todas las sociedades desde que éstas deben procurarse alimentos para sobrevivir (Abeyá Gilardon, 2016, p. 590; Aguirre, 2007). En Argentina pueden detectarse "experiencias en materia de asistencia alimentaria desde épocas muy tempranas" manifestadas en comedores escolares y ollas populares ya a fines del siglo XIX, siendo recién con la creación del Instituto Nacional de Nutrición a fines de la década de 1930 "que el Estado asume responsabilidad en esta materia, diseñando e implementando programas alimentarios que van evolucionando hasta generalizarse, hacia la década de 1970, la modalidad de distribución de alimentos" (Aulicino \& Díaz Langou, 2012: 14) ${ }^{3}$. No es hasta diciembre de 2002, con la creación del Programa Nacional de Alimentación y Nutrición (en adelante, PNAN) que "se comienza a esbozar en el país una política alimentaria" (Aguirre, 2004, p. 85).

La relación entre los programas alimentarios y el concepto de emergencia alimentaria se establece - por lo menos a nivel de justificación discursiva - a partir del acaecimiento de determinadas situaciones que dificultan el acceso a los alimentos, las cuales pueden presentar características externas - vg. catástrofes

3 El mencionado Instituto tiene como antecedente al Instituto Municipal de Enfermedades de la Nutrición, creado instancias del médico Pablo Escudero. Inaugurado en 1928, funcionaba dentro del Hospital Rawson y se dedicaba al estudio y asistencia de las enfermedades de la nutrición al inicio, luego fue incluyendo además de la dimensión asistencial, aspectos biológicos, químicos, económicos y sociales. “Entre 1934 y 1938 la suerte del establecimiento despertó el interés de autoridades políticas nacionales" y en 1938 fue convertido en Instituto Nacional de la Nutrición, mediante decreto del entonces presidente A. Justo (Buschini, 2015, p. 5). 
naturales o sociales - o inherentes a la persona - incapacidad sea tanto psíquica como física o material (de recursos materiales o financieros) - (CDESC, 1999, 15).

En Argentina, el abordaje político y legislativo de la cuestión del acceso a los alimentos a partir del concepto de emergencia alimentaria puede rastrearse a mediados de la década de 1980 y se extiende hasta la actualidad ${ }^{4}$. Esta prolongada extensión en el tiempo convoca a un replanteo del abordaje de los planes alimentarios limitado a contextos de emergencia, e incluso a un cuestionamiento más profundo acerca de los tratamientos legislativos que han recibido las situaciones calificadas como emergencias alimentarias.

La articulación entre emergencia y derecho humano a la alimentación se detecta a partir de la sanción de la citada Ley 25.724 en diciembre de 2002, la cual crea el PNAN. ${ }^{5}$ Dicha norma responde explícitamente al Dec. de necesidad y urgencia $N^{o} 108$ de enero de 2002. Este decreto declaraba la Emergencia Alimentaria Nacional hasta el 31 de diciembre de 2002 y ordenaba la creación del mencionado Programa destinado a la compra de alimentos, en el ámbito del entonces Ministerio de Desarrollo Social y Medio Ambiente.

4 Según Britos (2003, p. 23) la “creación del PAN en 1984 es de alguna manera el hito fundacional de la historia contemporánea de los programas alimentarios. Si bien el programa nace con carácter de emergencia y transitorio (la Ley establece dos años), continúa durante todo el mandato de Raúl Alfonsín y podríamos decir que con diferencias de nombres y modelos de gestión, continúa vigente hasta la actualidad".

5 La la ley 25.724 resultó del debate que se originó “en la opinión pública y algunas instituciones, oficiales y de la sociedad civil respecto de las políticas alimentarias" que requería la Argentina de entonces, en un contexto de marcada crisis económica y consecuente hambre en amplios sectores de la población (Britos et al, 2003, p. 35). El proyecto de ley se basó en una propuesta técnica realizada por el Grupo Sophia en conjunto con Poder Ciudadano y Red Solidaria, denominada "El Hambre más urgente", acompañada por una campaña del diario La Nación para reunir firmas y lograr el estatus de iniciativa popular (art. 39 CN). En julio de 2002 el Consejo Nacional de Coordinación de Políticas Sociales realizó “un Foro para un Plan Nacional de Alimentación y Nutrición, con la participación de 300 técnicos de organismos gubernamentales, legisladores, investigadores de universidades y centros de investigación, y miembros de organizaciones no gubernamentales" (Abeyá Gilardón, 2016, p. 597) cuyas conclusiones moldearon el texto luego promulgado en 2003. 
La Ley 25.724 menciona explícitamente el derecho humano a la alimentación (art. 1) aunque luego no lo retoma ni lo desarrolla. ${ }^{6}$ Si bien se reconoce el derecho de toda la ciudadanía a la alimentación, las medidas planteadas se limitan a un sector de la población considerado más vulnerable.

Aunque la incorporación de la perspectiva de los derechos humanos al tratamiento de la problemática del acceso a los alimentos representa una revisión del rol del Estado como agente principal en la gestión estratégica de medidas de garantías de los derechos humanos, su restricción a la situación de emergencia y al concepto de seguridad alimentaria, en la práctica limita el tratamiento de la cuestión alimentaria a un nivel básico de satisfacción de necesidades calóricas mínimas.

El concepto de seguridad alimentaria significa una ampliación del derecho a la alimentación respecto de su abordaje como acceso a un mínimo de calorías, al incorporar la idea de una alimentación adecuada en relación con los nutrientes necesarios para llevar adelante una vida sana y activa. $^{7}$ Sin embargo, la seguridad alimentaria no aborda la cuestión del control de los recursos, ni de los aspectos culturales y sociales de la alimentación.

La reciente Ley 27.519/19, que extiende la declaración de emergencia alimentaria de 2002 hasta 2022, retoma la perspectiva del derecho humano a la alimentación en relación a la problemática alimentaria. En el marco de esta última ley de 2019, se dicta la resolución 8/2020 del Ministerio de Desarrollo Social, mediante la cual se crea el Plan Argentina Contra el Hambre (en

\footnotetext{
6 Anteriormente el derecho a la alimentación se encontraba incorporado en el ordenamiento a partir de la recepción del PDESC en el Art. 75 inc. 22 de la CN. (Art. 11).

7 Según el informe de la Cumbre de la FAO sobre alimentación del año 1996 existe seguridad alimentaria "cuando todas las personas tienen acceso en todo momento a alimentos suficientes, seguros y nutritivos para cubrir sus necesidades nutricionales y las preferencias culturales para una vida sana y activa". Este concepto fue acuñado en 1973 para redireccionar los objetivos de la FAO, luego de dar por cumplidos aquellos por los que fue instituida en la posguerra, extendiendo su objetivo de lucha contra el hambre de Europa a todo el mundo (Cartwright Traylor, 1988, p. 187).
} 
adelante, PACH). Este Plan se apoya en el ya existente PNAN (Ley 25.724/02) y sobre todo otro programa institucional que lo complemente o tenga por fin dar respuesta a la cuestión alimentaria.

El presente trabajo se propone analizar los mecanismos de articulación entre el tratamiento de la emergencia alimentaria y el derecho humano a la alimentación que presenta el PACH. El análisis pretende señalar posibilidades de desarrollo del plan mediante sus vinculaciones (o ausencias de ellas) con otras normas existentes en el ordenamiento jurídico argentino vigente. Se parte de la siguiente hipótesis: la concentración de la política alimentaria nacional en programas de emergencia alimentaria significa una limitación del derecho humano a la alimentación a un aspecto básico (derecho a no morir de hambre) el cual, si bien es prioritario, no aborda la cuestión alimentaria en profundidad al deslindarla de otros aspectos sistémicos como son la producción, elaboración, distribución, venta y consumo de alimentos.

\section{Los niveles de protección del Derecho Humano a la alimentación}

El derecho humano a la alimentación presenta dos niveles de protección que se encuentran consagrados respectivamente en los párrafos uno y dos del Artículo 11 del Pacto de Derechos Económicos, Sociales y Culturales (PDESC). El primer párrafo refiere al derecho a la alimentación adecuada como parte del derecho a un nivel de vida digno. El segundo párrafo, en cambio, refiere a un nivel mucho más básico y primario de la alimentación que es el derecho a estar protegido contra el hambre, lo cual constituye además un presupuesto de cualquier alimentación adecuada.

Este nivel referente al hambre postula un derecho tan elemental que el PDESC lo califica como fundamental, siendo el único derecho en todo el Pacto que posee este calificativo. Tanto el Pacto de Derechos Civiles y Políticos (PDCP) como el PDESC recogen por única vez a este adjetivo en el artículo 5.2. refiriéndose a "los derechos fundamentales reconocidos en 
los Pactos". Pero lo hacen de manera genérica, en un sentido amplio, para abarcar a todos los derechos humanos. En cambio, el único derecho calificado individualmente como fundamental en ambos pactos es este segundo nivel del derecho humano a la alimentación en relación al hambre. ${ }^{8}$ Esta referencia a una fundamentalidad denota una urgencia particular que vuelve a este primer nivel ineludible, apremiante.

El derecho a estar protegido contra el hambre se satisface a través del acceso efectivo a alimentos que cubran un mínimo de calorías necesarias. En cambio, el derecho a la alimentación en su nivel más complejo, en tanto alimentación adecuada, hace referencia a un aspecto más integral y dinámico, que abarca elementos culturales y sanitarios y debe ser interpretado en el marco del derecho a un nivel de vida digno (Craven, 1995, p. 205; Haugen, 2007, p. 120). El derecho a una alimentación adecuada, en el sentido que le otorga FAO al concepto de seguridad alimentaria, implica el acceso a alimentos que permitan llevar adelante una vida activa y saludable. Esto hace referencia no sólo a las calorías sino también a los nutrientes y la calidad de los alimentos (CDESC, 1999, n. 6).

Un replanteo incluso de este segundo nivel se propone desde la perspectiva de la soberanía alimentaria. Este concepto refiere no sólo a la importancia del acceso, sino también del control de los recursos esenciales para la alimentación así como del proceso de producción de los alimentos, aspectos que hacen a la autonomía de los individuos y los pueblos en las decisiones vinculadas a su alimentación. Ello implica la promoción de las capacidades locales de autoabastecimiento, respeto a las formas locales de producción y consumo, así como a las costumbres y necesidades alimentarias (Quintana, 2008, p. 6).

Esta perspectiva se encontraba ya esbozada en el punto a) del segundo inciso del artículo 11 del PDESC, que relaciona la realización del derecho humano a la alimentación con la mejora

8 Este párrafo fue incorporado a propuesta de la FAO, que en 1963 lo presentó como parte de una campaña contra el hambre. Cf. Breining-Kaufmann, 1991, p. 62. 
de los métodos de producción, conservación y distribución de los alimentos. Sin embargo, el surgimiento del concepto de la soberanía alimentaria significó un movimiento del foco de la problemática desde la lucha contra el hambre hacia la lucha por una gestión colectiva recursos esenciales para la alimentación.

El enfoque de la soberanía alimentaria no sólo vino a complejizar y complementar el concepto de derecho humano a la alimentación, sino que aportó un disparador fundamental para un nuevo abordaje. El problema de la alimentación tiene que ver con una cuestión sistémica que abarca no sólo el acceso a los alimentos sino también la producción y el comercio, incluyendo el control y el dominio sobre los recursos esenciales para la alimentación.?

En este sentido, la perspectiva de la soberanía alimentaria pone en cuestión el abordaje lineal que venía gobernando las medidas de garantía del derecho a la alimentación. Estos abordajes lineales, que hacen referencia a una gradualidad o secuencialidad de las acciones, vienen justificando las medidas paliativas contra el hambre como "primera instancia" para luego afrontar otros niveles considerados más complejos de la problemática alimentaria.

\section{Los niveles de protección en el PACH}

El objetivo del PACH de abordar el problema de la alimentación desde un enfoque de los derechos humanos es explícito, así como el encuadre de este derecho desde la perspectiva de la soberanía alimentaria. Ello es reiteradamente enunciado en los considerandos y en los objetivos del Plan. El nivel de articulación entre esta perspectiva, los componentes del Plan y los

9 El concepto de soberanía alimentaria es postulado por La Vía Campesina - el principal gremio campesino a nivel global - como alternativa al concepto de seguridad alimentaria propuesto en la cumbre alimentaria de la FAO, poniendo el énfasis en la importancia del control sobre los recursos, en la participación y en la gestión de toda la cadena alimentaria, así como también en la importancia de las tradiciones campesinas para el sostenimiento de un modelo agroalimentario a largo plazo. Cf. Bonet de Viola, 2014. 
medios dispuestos para su implementación, es una cuestión que sólo podrá ser posteriormente analizada, una vez puesto en marcha. Por ello el presente análisis se limita a indagar tal articulación a nivel jurídico normativo.

El PACH es detallado en un Anexo a la resolución 8/2020 del Ministerio de Desarrollo Social de la Nación. Luego de describir sus fundamentos (introducción), objetivos general (I) y específicos (II), destinatarios (III) y modelo de gestión (IV), presenta en cinco componentes (incisos del punto V), las medidas concretas para su implementación, para finalmente bosquejar la modalidad de ejecución (VI). Los cinco componentes del PACH consisten en: A) Seguridad Alimentaria; B) Asistencia alimentaria en situaciones críticas o de emergencia; C) Apoyo a la producción y comercialización de alimentos; D) Fortalecimiento de redes comunitarias; E) Monitoreo y Evaluación. ${ }^{10}$

A continuación, se presentan las vinculaciones entre los niveles de protección del derecho humano a la alimentación y estos componentes, poniendo de relieve las tensiones, limitaciones y potencialidades del Plan. Este análisis se apoya en un relevamiento del conjunto normativo que contiene los diferentes programas alimentarios de las últimas cuatro décadas. La recuperación de estos textos permite observar ciertas lógicas normativas que los atraviesan y los limitan en sus alcances, lógicas regulatorias de las cuales el PACH es heredero.

\footnotetext{
10 Una de las grandes falencias señaladas en los planes alimentarios en Argentina fue su nula o deficiente evaluación (Abeyá Gilardon, 2016; Britos el al, 2003). El PACH incluye este aspecto como un componente, promoviendo para ello la aplicación de las ciencias y tecnologías. En este sentido, se lanzó una convocatoria a investigadores y tecnólogos para participar en el plan "Argentina contra el Hambre" lanzada en enero de 2020 desde el Ministerio de Ciencia, Tecnología e Innovación de la Nación, en conjunto con el Consejo Nacional de Coordinación de Políticas Sociales. En una primera instancia, se trata de reunir los resultados (parciales o finales) de proyectos y/o desarrollos tecnológicos y sociales "en problemáticas vinculadas con las acciones comprendidas en el mencionado Plan, para que sean incorporados al diseño de las políticas públicas en el corto plazo", a partir de su presentación voluntaria mediante un formulario. De: https://www.conicet.gov.ar/la-cienciay-la-tecnologia-se-suman-a-la-lucha-contra-el-hambre/
} 
En el primer apartado se analiza el nivel más básico del derecho humano a la alimentación y la lógica de linealidad que éste adquiere en su expresión normativa. En el segundo, se muestran otras lógicas más integrales de abordaje del derecho humano a la alimentación, las cuales aparecen también - pero en menor medida - en los textos normativos en relación al segundo nivel.

\section{III.1. Seguridad alimentaria: entre la linealidad subyacente y la declaración de emergencia}

La lógica de la linealidad que subyace a los programas de asistencia alimentaria se encuentra expresamente enunciada en los considerandos de la norma que a fines de 1980 crea el Bono Nacional Solidario de Emergencia (Dec. 400/89), uno de los tantos programas que el Poder Ejecutivo nacional diseñó para afrontar la problemática alimentaria. Se establece allí que "frente a la muerte de niños y la posibilidad de una hambruna generalizada, la sociedad organizada jurídicamente en el Estado no puede permanecer ajena y debe reconocerle al tema la prioridad que se merece, canalizando sus recursos en forma tal que se logre una solución temporal primero, y definitiva después, a través de la Revolución Productiva y la reforma social".

Este enfoque secuencialista, es de algún modo continuado en la Ley 25.724/2002 que crea el PNAN, en un contexto de declarada emergencia alimentaria (DNU 108/2002). Si bien cabe reconocer a esta norma el haber incorporado expresamente el derecho humano a la alimentación como fundamento para el tratamiento de la cuestión alimentaria, todavía lo hace enfocada en el primer nivel de este derecho humano (es decir, el referido al hambre) y limitando sus medidas a una parte de la población. Su decreto reglamentario (Dec. 1018/2003) considera que el PNAN "si bien surge para atender la situación de emergencia alimentaria de los sectores de la población más afectados, debería trascenderla y propender a elevar la calidad de vida de toda la población mediante el mejoramiento de su salud 
y nutrición, en el mediano y largo plazo" (considerandos del Dec. 1018/03).

Esta lógica gradualista se vuelve a reflejar en la falta de articulación entre los componentes A y B del PACH. Los incisos A y $\mathrm{B}$ reproducen las estrategias de los anteriores tratamientos de emergencia alimentaria ${ }^{11}$ : la ayuda alimentaria se traduce en el reparto de prestaciones alimentarias.

El inciso A, bajo el título de 'Seguridad Alimentaria', se ocupa principalmente del aspecto de la accesibilidad física o económica del derecho humano a la alimentación al incluir tres medidas: 1 . tarjeta alimentar, 2. prestaciones para comedores escolares y 3. prestaciones para merenderos y comedores comunitarios.

Es de resaltar la apelación al concepto de seguridad alimentaria en relación con la entrega de alimentos. Debe recordarse que, a los fines de cumplir con los postulados de este concepto (según la definición de FAO) la entrega de alimentos debería 'incluir todos los nutrientes necesarios para llevar adelante una vida sana y activa'. Una de las críticas a estos mecanismos directos reside en la falta de diversidad alimentaria y de micronutrientes en sus ofertas, sobre todo de parte de quienes afirman que incluso contando con los mismos recursos podría ofrecerse una alimentación de mejor calidad (O’Donnell y Britos, 2002; Abeyá Gilardón, 2016; Borrás y García, 2013). ${ }^{12}$

\footnotetext{
11 Para un análisis de los planes y programas alimentarios en Argentina, se pueden consultar, entre otros: Abeyá Gilardon (2016), Borrás \& García (2013), Britos et al. (2003), Maceira \& Stechina (2011), Sordini (2014), Teubal \& Palmisano (2013), Aulicino \& Díaz Langou (2012).

12 Durante los '90 "antes que programas de cobertura universal que atacaran las causas de la pobreza, se propiciaron programas focalizados apuntados a compensar las "faltas" (en nuestro caso el consumo) de los que quedaban fuera del mercado. Se propició la transferencia hacia los privados de los servicios para la población que pudiera pagarlos, mientras el Estado se reservaba la atención de los indigentes... Por otra parte, la insistencia en la ineficacia del Estado como administrador de la asistencia propició una gestión tercerizada (cuya eficiencia también está en duda) con programas contratados directamente con ONGs (civiles y confesionales) y organismos internacionales (...). Desde el punto de vista alimentario, entonces, tanto el mercado por la reducción de la capacidad de compra, como el Estado
} 
Esta asociación de la seguridad alimentaria con entrega de alimentos o dinero para su adquisición es uno de los principales puntos de crítica que la perspectiva de la soberanía alimentaria dirige al enfoque de la seguridad alimentaria (Vía Campesina, 2017). Se ha cuestionado que la noción de seguridad alimentaria puede coexistir con formas asistencialistas funcionales a un sistema de dominación y explotación monopolizada de los recursos esenciales para la alimentación (Shiva, 2003). Ello argumentado con discursos de solidaridad y beneficencia que terminan reforzando el sistema de jerarquías al cronificar situaciones de empobrecimiento y vulnerabilidad.

Antecedentes de la apelación a la solidaridad y el trabajo voluntario pueden rastrearse en las fundamentaciones de programas alimentarios anteriores. La Ley 23.056/84 que estableció el Programa Alimentario Nacional (en adelante, PAN) ordenaba que su aplicación debía "fomentar y orientar la solidaridad social, la donación de bienes y servicios del trabajo voluntario mediante la participación comunitaria y la difusión de los objetivos y contenidos" del mismo (art. 8). ${ }^{13}$ Más tarde, el Bono Nacional Solidario de Emergencia (Dec. 400/89) cuya fundamentación menciona las críticas al PAN, apela a la necesidad de "obviarse mecanismos burocráticos ineficientes y activar los resortes sociales derivados del principio de solidaridad". Con este argumento, el PEN resuelve implementar "sin ningún tipo de demoras un sistema que reúna las características de sencillez, eficacia y economía de costos", financiado con recursos genuinos y "los aportes solidarios de ciudadanos y entidades diversas" (considerandos del Dec. PEN 400/89) ${ }^{14}$. Se establecía

por la contracción e ideología en la inversión del gasto público y la negativa para intervenir en el mercado, contribuyeron a hacer más restringido el acceso a la alimentación de un creciente contingente de personas" (Aguirre, 2005, p. 6).

13 Una de las tres funciones a cargo la Comisión ejecutiva que administraba el PAN era "estimular la participación comunitaria y canalizar la solidaridad social para el desarrollo del programa" (art. 4).

14 Mediante este decreto se crea un Consejo Nacional para la Emergencia Alimentaria, presidido por el Ministerio de Salud y Acción Social y compuesto por los Ministros del 
incluso la posibilidad de invitar a los beneficiarios del régimen a prestar "su colaboración gratuita y solidaria para ayudar en la realización de tareas de interés comunitario" (art. 10).

El cuestionamiento de la apelación a la solidaridad frente a la situación de emergencia no apunta a la (noble, por cierto) iniciativa individual o colectiva frente a la calamidad ajena, sino a su institucionalización como parte de una política pública frente a una problemática tan compleja como la alimentación. Esta institucionalización de la solidaridad es funcional a un modelo asistencialista que no logra superar la lógica de linealidad.

Este esquema asistencialista se reproduce en el componente $\mathrm{B}$ del PACH (destinado a la 'Asistencia alimentaria en situaciones críticas y/o de emergencia') que refiere al reparto de prestaciones alimentarias en forma directa o a través de organismos gubernamentales o no gubernamentales. A través de la entrega de lotes de alimentos a la población en situación de vulnerabilidad y urgencia, este componente responde directamente al primer nivel del derecho humano a la alimentación, constituyéndose en el principal exponente del abordaje lineal de la emergencia alimentaria en el Plan.

Estas medidas se asumen como politicas de emergencia pretendiendo atender a la urgencia que denota la fundamentalidad del derecho a la alimentación referido al hambre. De allí viene fundamentada la inmediatez y la consecuente necesidad de medidas inmediatas, a corto plazo y focalizadas en la población vulnerable. Es así que el enfoque de emergencia habilita el tratamiento de la problemática alimentaria como una cuestión coyuntural, paliativa.

\footnotetext{
Interior, y de Trabajo y Seguridad Social, un representante de las Fuerzas Armadas, un representante de la Iglesia Católica, un representante de la Confederación General del Trabajo y un representante de las organizaciones empresarias de la industria y el comercio. Tenía por función la promoción de "la movilización solidaria para la recaudación de fondos" y la auditoría de la aplicación de estos fondos a los programas de emergencia social (art. 3). En el orden provincial y municipal se debían constituir este tipo de Consejos de Emergencia, replicando el esquema de composición. Los consejos locales tendrían a su cargo la dirección y fiscalización en sus jurisdicciones el cumplimiento de los objetivos del sistema.
} 
En la experiencia argentina, esto se viene reflejando en el lanzamiento de programas alimentarios dictados en el marco de declaraciones de emergencia. La actual declaración de "emergencia alimentaria" puede reconstruirse jurídicamente en una cadena normativa que va desde el decreto de necesidad y urgencia 108/2002 a la Ley 27.519 de 2019. Es decir, según la propia resolución ministerial que establece el PACH, la extensión temporal de la emergencia alimentaria es de veinte años. Esto habilita el interrogante acerca de si el lapso de veinte años puede calificar como situación de excepción o se trata de un emergente sistémico que requiere una revisión profunda del sistema de producción, elaboración, distribución, venta, publicidad y consumo de los alimentos.

La limitación de los tratamientos de emergencia a medidas cortoplacistas y destinadas a la población vulnerable se puede observar en casi toda la regulación de los programas alimentarios argentinos anteriores al PACH que se enfocan en el suministro directo de alimentos y la entrega condicionada de dinero principalmente a los grupos más afectados (Maceira, 2010). Así, el PAN (Ley 23.056/84) justificaba la delegación de facultades al poder ejecutivo nacional para su realización en la "crítica situación de deficiencia alimentaria aguda de la población más vulnerable y de pobreza extrema" (art. 1).

En contexto de hiperinflación, el decreto 400/89 (Bono Nacional Solidario de Emergencia) se dicta considerando que la tasa de desocupación era "la más alta de los últimos cincuenta años" y gran parte de la población estaba "sumida en la miseria que implica no alcanzar los mínimos necesarios para la subsistencia". Así, las medidas dispuestas debían regir "de inmediato puesto que no se puede eludir la toma de decisiones cuando se está frente a la degradación impuesta por la miseria y cuando el hambre amenaza a millares de compatriotas"15.

\footnotetext{
15 A diferencia del PAN, este Decreto se dictó (el 1/8/1989) sin una previa ley que le delegara las competencias reglamentarias, invocando las facultades emergentes por el artículo 86, inciso 1, de la Constitución Nacional por entonces vigentes. A poco tiempo se
} 
En el 2000, reconociendo que para entonces la "superposición de programas, la duplicación de esfuerzos y los altos costos operativos, no garantizan en forma suficiente el complemento alimentario a la población en situación de pobreza crítica" y que era "prioritario establecer un sistema de seguridad alimentaria para los sectores más vulnerables del país", se crea el Sistema Alimentario Federal (Dec. PEN 547/2000) que integraba los programas existentes. Este Sistema fue dejado sin efecto dos años más tarde (Dec. 328/2002), invocando "las limitaciones presupuestarias y financieras existentes" y la necesidad de "optimizar los escasos recursos disponibles, y no superponer acciones programáticas" 16 .

La principal dificultad que presenta la vinculación de la cuestión del hambre con el contexto de emergencia consiste en la limitación de las acciones a paliativos, obstaculizando la mirada a largo que requieren las medidas integrales. Las estrategias de emergencia suelen obviar las múltiples facetas que abarca la problemática de la alimentación en su complejidad, incluyendo la llamada "doble carga" de la malnutrición (Fanzo, 2016). ${ }^{17}$ Ésta exige ser abordada como un problema sanitario vinculado con el consumo de productos alimenticios ultraprocesados (Barruti, 2013). Ello implica a su vez comprender las conexiones de este consumo con la homogeneización y estandarización de la alimentación en un contexto de comercio global (Fischler, 2010),

sancionaron dos leyes relacionadas: la Ley 23740 (del 28 de septiembre de 1989) que creaba el Programa de Emergencia Social, estableciendo una "contribución solidaria" (un impuesto) para su financiamiento y la Ley 23767 (del 27 de diciembre de 1989) que implementa un Programa de Políticas Sociales Comunitarias (POSOCO) "destinado a atender las necesidades alimentarias, sanitarias, asistenciales, habitacionales y/o locativas de los sectores sociales más carenciados del país".

16 Al discontinuar el Sistema Alimentario Federal, se deja sin efecto el Programa de Apoyo Familiar Unidos (Decreto PEN N 547/2000) pero se exceptúa de esa derogación general al Pro-Huerta, que continuaba funcionando en la órbita del INTA.

17 La noción de malnutrición incluye no solo el problema de la desnutrición, sino la también del sobrepeso, que involucra una serie de enfermedades no transmisibles derivadas de una alimentación deficiente (diabetes, obesidad, hipertensión y demás problemas circulatorios y cardíacos). 
la desmaterialización de los sistema alimentarios (Filardi y Prato, 2018), las agresivas estrategias de publicidad de estos alimentos (Nestle, 2007), las largas cadenas de intermediarios y la hegemonía del supermercadismo y las corporaciones que los monopolizan (Teubal \& Rodríguez, 2002).

Por su parte, mecanismos paliativos como la entrega de tarjetas alimentarias o entrega directa de alimentos, a la vez que contribuyen a la estigmatización de sus usuarios (Pomar y Tendero, 2016, p. 40) invisibilizan aspectos culturales de la alimentación, como la comensalidad y los hábitos alimentarios, reforzando los inequitativos patrones alimentarios preexistentes (Borrás y García, 2013).

Por todo ello, la coyunturalidad de las respuestas que los componentes A y B ofrecen pueden entrar en conflicto con la integralidad del abordaje que promete el PACH en sus considerandos iniciales. La principal dificultad reside en considerar las medidas para combatir el hambre como un "primer paso" para la resolución del problema alimentario. En la medida en que este primer paso esté compuesto por acciones asistencialistas que cronifican las situaciones de empobrecimiento y dependencia (Pomar y Tendero, 2016, p. 40) sólo se refuerza un sistema de producción y un mercado globalizado de industrias alimentarias concentradas (Teubal y Palmisano, 2013) contribuyendo a perpetuar el sistema de apropiación y gestión de los recursos en el cual reside el propio problema.

\section{III.2. Hacia la autoproducción y la soberanía alimentaria}

El PACH incorpora la perspectiva de la soberanía alimentaria, aunque en forma algo inarticulada y a partir de una definición de fuente extranjera, lo cual demuestra cierta desatención del origen del término y la producción local de conocimientos sobre la cuestión. ${ }^{18}$ De cualquier manera el texto de la norma

18 Concretamente la Resolución cita (nota al pie núm. 5) como fuente de referencia del concepto de Soberanía alimentaria un texto en inglés obtenido desde la web de una Fundación 
refiere acertadamente, ya en sus considerandos, a la importancia de un enfoque integral de la problemática alimentaria, al vincularlo a soberanía alimentaria y otras dimensiones como la economía, el cuidado, la salud y la educación.

La centralidad que procura darse al enfoque de la soberanía alimentaria puede desprenderse de su incorporación en el objetivo general del PACH. Esta centralidad se replica en los objetivos específicos relativos a la "participación de las familias y las comunidades en la producción de alimentos a fin de diversificar y mejorar sus consumos en pos de una alimentación saludable", la promoción de "sistemas de producción de alimentos a través del fortalecimiento de la economía solidaria, social y popular, el cooperativismo y la agricultura familiar" (punto II del Anexo, Res. 8/2020).

Este enfoque es retomado en los componentes $\mathrm{C}$ y $\mathrm{D}$ del PACH. En sintonía con los considerandos y objetivos, estos componentes refieren a aspectos más complejos de la alimentación que los anteriores (A y B). ${ }^{19}$ El principal potencial de este Plan reside en esta atención a la complejidad que amerita la implementación del derecho humano a una alimentación adecuada. Sin embargo, quedará por ver el grado de traducción de tal

Canadiense. Teniendo en cuenta que el concepto surge de la organización "La Vía Campesina", una referencia a los textos provenientes de ésta hubiera sido preferible. También existen textos de investigadores locales que dan cuenta del tema desde una perspectiva más cercana a la realidad argentina, así como la producción de movimientos sociales. En este último sentido, se recupera la documentación producida en el marco del el Primer Foro por un Programa Agrario Soberano y Popular Buenos Aires, realizado 7 y 8 de mayo de 2019 en Buenos Aires. Disponible en: http://foroagrario.org/documentos/

19 Según consta en el apartado V del Anexo de la Res. Min. DS 8/2020: “C. apoyo a la producción y comercialización de alimentos: El objetivo de este componente es favorecer la producción y comercialización de alimentos de la economía solidaria, social y popular, el cooperativismo y la agricultura familiar. Este componente incluye: 1. Creación y fortalecimiento de mercados populares. 2. Financiamiento a productores de la economía solidaria, social y popular. 3. Apoyo a la producción de la agricultura familiar. D. Fortalecimiento de redes comunitarias: El objetivo de este componente es fortalecer las redes de actores comunitarios para que operen de manera integrada en el fortalecimiento de las familias en el cuidado de la primera infancia, la seguridad alimentaria, la salud y la educación". 
integralidad en la articulación efectiva que tengan en su implementación los diversos componentes del PACH como medidas de garantía del derecho humano a la alimentación.

La articulación entre las medidas relativas al primer nivel de protección del derecho a la alimentación (lucha contra el hambre) y la producción y comercialización de los alimentos, encuentra pocos antecedentes en la normativa de emergencia alimentaria previa. En la Ley 23.056/86 que creó el PAN, pueden encontrarse referencias significativas. Se establecía allí que se debía dar "prioridad a los productos regionales, que se adecuen al Programa Alimentario Nacional y su producción sea local" (art. 11, inc. a) en los mecanismos de producción, compra y distribución de alimentos para el programa y se debía propiciar "el estímulo de la alimentación natural" y promover la lactancia materna (art. 11, inc. d). Sin embargo, a juzgar por críticas posteriores, estos propósitos del PAN no fueron alcanzados ${ }^{20}$.

En relación a la implementación de acciones por la soberanía alimentaria, es de destacar el programa Pro Huerta, tanto por sus medidas como por su estabilidad en el tiempo. Este programa fue aprobado por Res. $N^{\circ}$ 239/90 INTA, más tarde integrado como componente del PNSA, en el marco de la Ley $\mathrm{N}^{\mathrm{o}}$ 25.724/02. Se trata de un programa del Instituto Nacional de Tecnología Agropecuaria (organismo perteneciente al Ministerio de Agricultura), en vinculación con el Ministerio de Salud y Desarrollo Social. Está destinado al "impulso productivo a través de la implementación de huertas que por medio de la entrega de insumos agropecuarios, herramientas, capacitaciones y asesoramiento busca apoyar la producción de frutas y verduras" y también promueve la comercialización de la

20 El PAN se caracterizaba por el reparto de alimentos secos. Según Britos (2003, p. 23), "pocos programas tuvieron una administración tan centralizada como el PAN. El Gobierno Nacional, por medio de la ex Junta Nacional de Granos, adquiría los alimentos que se embalaban en dos plantas, una en Buenos Aires y la otra en Entre Ríos para ser distribuidas a todas las provincias. (...) El PAN resultó un programa básicamente asistencialista, cuestionado en reiteradas ocasiones por su uso clientelar y prácticas poco transparentes en los procesos de adquisición de alimentos". 
producción. Tiene como destinatarios a la población en situación de vulnerabilidad: niños en escuelas de áreas críticas, con prioridad de aquéllas con comedor escolar o en áreas económicamente deprimidas, marginales o rurales dispersas, con una cobertura geográfica urbana y rural ${ }^{21}$. Puede considerarse que en sus treinta años de existencia, este programa ha cumplido un rol central en el desarrollo de capacidades de las poblacione destinatarias así como en la promoción de la agroecología, en la medida que fue "rápida y crecientemente apropiado por el conjunto de actores implicados (Huerteros, promotores, técnicos e instituciones vinculadas) y se constituyó en una experiencia a gran escala de desarrollo de capacidades sociorganizacionales y productivas, atravesadas a su vez por los valores de la solidaridad y la cooperación" (Cittadini, 2014, p. 122).

En la Ley 25.724/02, que crea el PNSN, se detectan algunas referencias indirectas a estos aspectos. Esta norma ordenaba la creación de una red de comisiones de nutrición y alimentación (una nacional y una serie de comisiones provinciales y municipales similares) para la coordinación del programa (art. 4). Según el texto de esta ley, las comisiones provinciales tienen, entre otras funciones, las de "estimular el desarrollo de la producción alimentaria regional a fin de abastecer de los insumos necesarios a los programas de asistencia alimentaria locales, respetando y revalorizando la identidad cultural y las estrategias de consumo locales" (art. 7 inc. d); "impulsar la generación de políticas de abastecimiento alimentario en los niveles locales a fin de garantizar la accesibilidad de toda la población, especialmente a los grupos mencionados en el artículo $1^{\circ} \mathrm{y}$ promover la creación de centros de provisión y compra regionales (art. 7 inc. e); "promover la organización de redes sociales posibilitando el intercambio dinámico entre sus integrantes y

21 De: https://plataformacelac.org/programa/283 El programa se apoyó en alianzas con ONGs que anteriormente desarrollaban este tipo de acciones en diferentes territorios, colaborando para que éstas puedan acceder no solo a capacitación en una metodología de trabajo eficiente sino también a recursos materiales. 
con los de otros grupos sociales, potenciando los recursos que poseen" (art. 7 inc. f). No obstante, como se explicará en el punto siguiente, no hay registro de funcionamiento alguno de esta red de coordinación interjurisdiccional de las comisiones de alimentación y nutrición del PNSA.

Más recientemente, puede rastrearse una regulación de la producción de alimentos directamente vinculada con la noción de Soberanía Alimentaria en la Ley 27.118/2014 de Agricultura Familiar. Esta ley declara de interés público la Agricultura Familiar, Campesina e Indígena y crea un Régimen de Reparación Histórica. Esta ley contiene, entre otras, medidas para el "acceso a la tierra para la agricultura familiar, campesina e indígena, considerando la tierra como un bien social" (art. 15); crea un Banco de Tierras para la Agricultura Familiar (art. 16); arbitra su forma de adjudicación (art. 17) y establece un programa para la regularización dominial de tierras de la agricultura familiar, campesina e indígena (art. 18). Sin embargo, esta norma no fue aún reglamentada, no pudiéndose a implementar en consecuencia tales medidas. ${ }^{22}$

Es decir, los antecedentes normativos vienen demostrando que la sola incorporación de perspectivas vinculadas a la soberanía alimentaria en los textos legales no garantiza la traducción de esas intenciones en medidas concretas que la implementen, en el sentido de avanzar hacia la garantía del derecho humano a la alimentación. La perspectiva de la soberanía alimentaria exige una visión sistémica que no puede restringirse a medidas aisladas.

\footnotetext{
22 También ordena a la autoridad de aplicación (El Ministerio de Agricultura, Ganadería y Pesca, art. 9) impulsar la realización de ferias locales, la promoción de marcas comerciales, de mecanismos de certificación, la compra bajo "prioridad absoluta en la contrataciones directas que realice el Estado nacional para la provisión de alimentos" (art. 22). Dispone reglas sobre desarrollo tecnológico, asistencia técnica e investigación (arts. 23 a 26), infraestructura y equipamientos rurales (arts. 29 y 30 ) y crea diversos instrumentos de promoción vinculados a sanidad agropecuaria, beneficios impositivos, régimen previsional especial, certificaciones, seguro integral para la agricultura familiar y créditos (art. 32).
} 


\section{Observaciones de técnica legislativa y legitimidad democrática}

La desarticulación sustantiva entre la lógica sistémica que exige la perspectiva de la soberanía alimentaria y la coyunturalidad propia de los mecanismos de emergencia que se encuentra en el PACH, se refleja también en las cuestiones procedimentales relativas a la técnica jurídica utilizada. En efecto, la complejidad e integralidad que implican los postulados de la soberanía alimentaria entran en contradicción con el carácter acotado del ámbito de competencia formal del Plan regulado en una única órbita ministerial. Es decir, la expresa aspiración del PACH de convertir la lucha contra el hambre en 'política de estado' no se condice con la jerarquía normativa del dispositivo jurídico por el cual éste se instrumenta, verbigracia un anexo a una resolución ministerial del área de Desarrollo Social.

Si bien en principio no es plausible que un Ministro ejerza potestades reglamentarias (es decir, el dictado de normas generales y abstractas) se reconoce que, más allá del art. 103 de la Constitución nacional ${ }^{23}$ "existe un fenómeno de dispersión subjetiva de la potestad regulatoria reglamentaria en los Ministros y demás órganos inferiores de la Administración" (Balbín, 2015, p. 163). La fuente normativa de estas resoluciones que exceden las cuestiones económicas y administrativas del propio Ministerio (como parece ser el caso del instrumento normativo que contiene el PACH) emanaría de la competencia derivada por transferencia mediante normas específicas.

En el caso de la resolución Ministerial 8/2020, esta transferencia opera por delegación o desconcentración de poder reglamentario del presidente según Ley de Ministerios $\mathrm{N}^{\circ} 22.520 / 81$ que atribuye al Ministerio de Desarrollo social la competencia

\footnotetext{
23 Según el artículo 103 de la Constitución Nacional (CN) "Ios Ministros no pueden por sí solos, en ningún caso, tomar resoluciones, a excepción de lo concerniente al régimen económico y administrativo de sus respectivos departamentos".
} 
para la asistencia de los sectores más vulnerables. ${ }^{24}$ Esta competencia es reforzada en la referencia que los fundamentos de la propia resolución hacen a la situación de emergencia, particularmente en relación con la Ley de Emergencia Alimentaria $\mathrm{N}^{\mathrm{o}} 27.519 / 19 .{ }^{25}$ Es decir, la habilitación de la competencia del Ministerio de Desarrollo Social en la problemática alimentaria se fundamenta en la situación de emergencia y su vinculación con la población vulnerable.

Ello sin embargo encuentra cierta contradicción en las sucesivas reiteraciones de los considerandos de la propia resolución 8/2020 respecto de la necesidad de "políticas y estrategias" que sean "integrales y transversales", basadas "en un enfoque interdisciplinario garantizando la coordinación interjurisdiccional e intersectorial de diversos organismos y niveles del Estado" y la sociedad civil. Expresamente se reconoce que "resulta necesario implementar acciones que, con la participación de los distintos actores de la sociedad, nos permitan dar respuesta a las demandas de la población destinataria de las politicas de Estado que se llevan a cabo en cumplimiento de las competencias de esta Cartera Ministerial'. Si la 'población destinataria' de las políticas que hacen a las competencias de esa cartera se reduce a 'los sectores más vulnerables' (tal como la misma norma expresa) y la colaboración en el cumplimiento de los tratados internacionales

24 Esto se explica en los considerandos de la Res. que invocan la distribución de competencias de la Ley de Ministerios № 22.520 y sus normas modificatorias y complementarias. Según esta última, "compete al Ministerio de Desarrollo Social asistir al Presidente de la Nación y al Jefe de Gabinete de Ministros en todo lo inherente a la política social orientada a la asistencia, promoción, cuidados e inclusión social, desarrollo humano, seguridad alimentaria, reducción de la pobreza, y desarrollo de igualdad de oportunidades para los sectores más vulnerables, en particular para las personas con discapacidad, las niñas, niños y adolescentes, las mujeres y los adultos mayores, la protección de las familias y el fortalecimiento de las organizaciones comunitarias, así como en lo relativo al acceso a la vivienda y el hábitat dignos, y al cumplimiento de los compromisos asumidos en relación con los tratados internacionales y los convenios multinacionales, en materia de su competencia" (resaltado de las autoras).

25 Se enuncia allí que "en virtud de la emergencia alimentaria y nutricional por la que atraviesa nuestro país, resulta imprescindible entender que todas las acciones y estrategias que lleva adelante este Ministerio... tienen como finalidad última brindar respuesta a tan angustiante situación". 
se limita a la 'materia de su competencia', entonces este PACH dictado mediante resolución no podría considerarse una acción en pos de la garantía del derecho humano a la alimentación de la ciudadanía toda, con enfoque integral. Es decir, no podría constituirse como una medida capaz de abordar la complejidad de la problemática alimentaria.

Debido al alcance de las acciones propuestas en el desarrollo del PACH que, tal como se analizó, exceden las medidas de coyuntura para la población vulnerable, y el amplio rango de temas que aspira a cubrir, el tipo y rango normativo elegido para su dictado (resolución ministerial) es significativamente modesto.

Sería técnicamente más conveniente que una apuesta política de estas dimensiones fuera implementada mediante una constelación normativa que tuviera como eje una ley nacional que dialogue con la regulación existente sobre las temáticas conexas $\mathrm{y}$, por sobre todo, se oriente al cumplimento de los derechos humanos reconocidos constitucionalmente para toda la población. En lo que hace a la faz administrativa, correspondería luego reglamentar esta ley por decreto y dictar eventuales desarrollos complementarios en una o más resoluciones ministeriales, según las distintas jurisdicciones involucradas.

En dirección al formato de ley nacional como instrumento normativo más adecuado, se dirige la recomendación del Parlatino para fortalecer el desarrollo institucional de lucha contra el hambre del continente. Desde ese ámbito, se diseñó con ese objetivo una Ley marco sobre Derecho a la Alimentación, Seguridad y Soberanía alimentaria. ${ }^{26}$ En esta línea se destaca el antecedente brasileño del Proyecto Hambre Cero (Fome Zero), cuyo éxito tuvo como gran referencia una comisión parlamentaria. ${ }^{27}$

\footnotetext{
26 Aprobada en la XVIII Asamblea Ordinaria del Parlamento Latinoamericano, 30 de noviembre al 1 de diciembre de 2012, Panamá. Como autor del Proyecto de Ley Marco es reconocido el diputado uruguayo José Carlos Cardoso, Presidente de la Comisión de Agricultura, Ganadería y Pesca del Parlatino (Parlamento Latinoamericano \& FA0, 2012, p. 5)

27 En este sentido, el citado diputado uruguayo autor de la Ley marco advertía, que el proyecto brasileño "tenía también su reflejo dentro del parlamento, no era solamente un desafío del
} 
El involucramiento del Congreso responde a la necesaria ampliación de legitimidad democrática que en un sistema republicano exige las magnitud de las acciones que implican un Plan de estas características. El consenso político plural que supone el proceso de debate y aprobación de una ley le otorga a sus medidas mayores posibilidades de estabilidad que las dispuestas mediante instrumentos administrativos delegados. Si bien el tiempo que implican los procesos de debate legislativos podría entenderse como un obstáculo para una temática de esta relevancia, la complejidad de la problemática alimentaria ameritaría un debate de esta envergadura.

Un enfoque integral como el que apela el $\mathrm{PACH}$ requiere un diseño institucional efectivo que permita articular las acciones de lucha contra el hambre con una estrategia nacional de regulación en materia de agroindustria y economía, relativas a la producción, distribución, venta y consumo de alimentos del país. ${ }^{28}$ Discursivamente, ello implicaría la articulación de los niveles de emergencia y soberanía. Técnicamente, precisaría del diseño de un plan de retroalimentación entre las carteras ministeriales temáticamente vinculadas, procurando no generar contradicciones internas en el propio ordenamiento que puedan traducirse en limitaciones a la efectividad del PACH. Esto aplica tanto para la articulación "interna" entre los actores estatales, como para la articulación "externa" con el sector privado vinculado al mercado.

De hecho, la vigente Ley 25.734/02 (que crea el SNSA) y su decreto reglamentario, contienen un dispositivo de gestión

\footnotetext{
gobierno y del ministro de turno, era un desafío del país y para que sea del país el Parlamento tiene que estar involucrado" (Cardoso, 2015).

28 En lo que respecta a la conexión de la problemática alimentaria con la regulación de lo económico y la cuestión agraria, los fundamentos normativos del PACH, expresados en los considerandos de la Res. Ministerial 8/2020, vinculan la problemática del hambre con el derecho humano a la alimentación reconocido constitucionalmente y se hacen cargo de la importancia de cumplir con los compromisos internacionales asumidos al respecto. Reconocen también la necesidad de políticas integrales y de la colaboración interjurisdiccional. Sin embargo, es notable la ausencia de vinculación expresa con la regulación de los aspectos económico-técnicos de la seguridad alimentaria.
} 
y coordinación interministerial para la seguridad alimentaria en su aspecto de acceso a los alimentos ${ }^{29}$. Esta ley ordenaba la creación de una Comisión Nacional de Nutrición y Alimentación (en adelante, CNNA) para la coordinación del Programa, además de comisiones provinciales y municipales similares (art. 4). ${ }^{30} \mathrm{Sin}$ embargo, no hay registro en los sitios webs de las áreas involucradas que esta CNNA haya sido puesta en funcionamiento ${ }^{31}$.

29 Aún encuadrado dentro de la lógica de la declaración de emergencia y destinada a una población vulnerable focalizada, el Plan Nacional de Seguridad Alimentaria fue superador en este aspecto de técnica legislativa, ya que fue lanzado mediante una ley del congreso ( $N^{0} 25.724$ sancionada el 27 de diciembre de 2002) reglamentada por Decreto 1018/2003. A diferencia de planes alimentarios anteriores (cuya ejecución estaba a cargo de la cartera de desarrollo social o de salud) la autoridad de aplicación es ejercida conjuntamente por los Ministerios de Salud y de Desarrollo Social (art. 3, Ley 25724).

30 La CNNA tiene amplias funciones: diseñar estrategias para la implementación, difundir el programa, fijar mecanismos de control, criterios de acceso al programa, etc. En el decreto reglamentario se detalla su composición (miembros designados por el PEN, bajo régimen ad honorem): representantes de los ministerios de Desarrollo Social (2), Salud (1), Educación, Ciencia y Tecnología (1), Economía y Producción (1), Trabajo, Empleo y Seguridad Social (1), Planificacion Federal, Inversion Publica y Servicios (1), pudiendo invitarse hasta cuatro "Organizaciones No Gubernamentales de mayor representatividad debidamente acreditadas y con experiencia de gestión en el área" (reglamentación del art. 4, en Decr. 1018/03).

31 Confusamente, una entrada de la página del Ministerio de Desarrollo Social (https:// www.argentina.gob.ar/noticias/mesa-de-coordinacion-de-politicas-alimentarias-ysaludables Acceso: 11/02/2020) sobre una reunión del Consejo Nacional de Coordinación de Políticas Sociales en 2017, linkea bajo la denominación Comisión Nacional de Nutrición y Alimentación del Programa de Educación Alimentaria Nacional (Decreto 1018/03) enmarcada en la Ley 25.724 (Programa de Alimentación y Nutrición Nacional) con el sitio de la Comisión Nacional de Alimentos (CONAL http://www.conal.gob.ar/) un organismo diferente, enmarcado en otra legislación (Decreto 815 de 1999) que se encarga del asesoramiento, apoyo y seguimiento del Sistema Nacional de Control de Alimentos que tiene por fin la aplicación y actualización del Código Alimentario Argentino (Marichal, 2016). En marzo de 2013, desde el Congreso se solicitó al PEN el cumplimiento de la Ley 25724. Entre otras cuestiones, se solicitaba informar "si se encuentra funcionando la Comisión Nacional de Nutrición y Alimentación Nacional creada por el Artículo 4 de la Ley 25.724, teniendo en cuenta la representación de las organizaciones no gubernamentales como lo define el inciso a del citado artículo" y que se detalle qué acciones se están realizando "para el fortalecimiento de la producción alimentaria regional, la autoproducción y el fortalecimiento del comercio de cercanía, respetando y valorizando las culturas y estrategias de consumo locales". Proy. de Res. Expediente 0694-D-2019, disponible en: https://www.diputados.gov.ar/proyectos/ proyecto.jsp?exp=0694-D-2019. 
La experiencia regulatoria en materia de seguridad alimentaria, en su aspecto de inocuidad, muestra que es factible poner en práctica una regulación interjurisdiccional, horizontal y verticalmente. Así lo demuestra la coordinación interministerial (salud y agricultura) de la autoridad de aplicación del Código Alimentario argentino (en adelante, CAA) ${ }^{32}$. La coordinación está regulada por un Sistema Nacional de Control Alimentario creado mediante Decreto $N^{\circ}$ 2194/94. (modif. por Dec. 815/99). Este sistema articula diversas reparticiones estatales centrales en la gestión de la cuestión alimentaria a través de Comisión Nacional de Alimentos (CONAL), ${ }^{33}$ la cual a su vez está compuesta por representantes de la ANMAT, el SENASA y las autoridades de aplicación sanitaria-alimentaria de CABA y las provincias ${ }^{34}$. La funcionalidad y eficacia de este complejo esquema institucional demuestra la viabilidad de una articulación de estas características.

Si el PACH es sustantivamente comprendido como un plan alimentario integral y coordinado, en materia de implementación y monitoreo corresponde la articulación de sus componentes con el engranaje institucional a cargo de la aplicación y actualización de las disposiciones del CAA ${ }^{35}$. Es decir, el Ministerio de Desarrollo Social - eje del PACH - debería convocar

32 Este código contiene la regulación del aspecto higiénico-bromatológico y comercial de la seguridad alimentaria. Ley 18.284 de 1979, Dec. reglamentario, normas comp. y modif. http://www.conal.gob.ar/CAA.php Desde 1994 se estableció que cualquier modificación de este Código debía emitirse mediante resoluciones ministeriales conjuntas.

33 El SNCA está integrado por la CONAL, el SENASA y la ANMAT. Las Autoridades Sanitarias Provinciales y de CABA son invitadas a integrarse (art. $4^{\circ}$, Dec. 815/99).

34 Esta comisión actúa en la órbita del Ministerio de Salud y está encargada de las tareas de asesoramiento, apoyo y seguimiento del SNCA (art. $5^{\circ}$, Dec. 815/99). Para mayor detalle sobre su composición actual, consultar: http://www.conal.gob.ar/representantes.php (Acceso: 04/02/2020). Sus decisiones se basan en los dictámenes de grupos de trabajos compuestos por expertos. http://www.conal.gob.ar/grupos_trabajo.php

35 Se trata, en definitiva, de las normas a las que deben sujetarse "toda persona, firma comercial o establecimiento que elabore, fraccione, conserve, transporte, expenda, exponga, importe 0 exporte alimentos, condimentos, bebidas o primeras materias correspondientes a los mismos y aditivos alimentarios" (art. 1 del CAA). 
para su implementación a los organismos encargados de gran parte de la regulación de la seguridad alimentaria en Argentina, directamente o través de la CONAL.

\section{Perspectivas}

El derecho humano a la alimentación significa ante todo la prerrogativa de todo ser humano de poder alimentarse a sí mismo y a la propia familia y, sólo en caso de necesidad, a recibir directamente alimentos (ONU/FAO, 2010, p. 4). En este sentido refiere el CDESC a las obligaciones del Estado respecto al cumplimiento del derecho humano a la alimentación, determinando que no sólo debe respetarlo, evitando interferir en su pleno goce, protegerlo - evitando que otros interfieran - sino ante todo garantizarlo. La obligación primordial del Estado consiste, además de respetar las fuentes de alimentación (agricultura, pero también fuentes de trabajo) y proteger de amenazas externas aquellas fuentes, consiste en promover y generar una situación en la que cada uno pueda alimentarse a sí mismo y a la propia familia.

La principal problemática de los planes de emergencia alimentaria en Argentina, teniendo en cuenta que se han extendido por veinte años, reside en que las medidas paliativas se han consolidado, volviendo a la asistencia un régimen estabilizado. Ello no sólo ha contribuido a desviar cualquier intento de fortalecimiento de la producción alimentaria local y alternativa - como las medidas de la Ley de Agricultura Familiar - sino que ha reforzado un sistema de dependencia y jerarquización ${ }^{36}$.

El lanzamiento del PACH significa una demostración de cierta intencionalidad política hacia un tratamiento integral de la

\footnotetext{
36 Esto pone de relevancia una cuestión no menor respecto del rol del Estado como garante del derecho humano a la alimentación: la dinámica de afianzamiento de las jerarquías sociales es reproducida no sólo en el ámbito privado del mercado, sino también por la propia estructura del Estado a través de sus mecanismos jurídicos de producción y aplicación normativa centralizada (Mattei, 2013, p. 31).
} 
problemática alimentaria. La incorporación de la perspectiva de soberanía alimentaria y el reconocimiento explícito de la complejidad de la cuestión, representan el principal potencial del PACH frente al derecho humano a una alimentación adecuada.

Sin embargo, este enfoque integral, que reclama medidas a largo plazo para toda la población y a partir de la complejidad que amerita la multidimensionalidad de la seguridad alimentaria, colisiona en la propia norma con el enfoque de emergencia que viene gobernando las estrategias nacionales frente a las situaciones de crisis alimentarias. Esta estrategia involucra el dictado de medidas de coyuntura, la concentración de la asistencia dirigida a la población vulnerable, y la limitación de las acciones a la facilitación del acceso a prestaciones alimentarias.

Uno de los grandes desafíos que presenta el PACH consiste en la articulación de lo enunciado en los considerandos, los objetivos y en los componentes referidos a la emergencia (A y B) con aquellos referidos a la soberanía alimentaria (C y D). Será en su posterior reglamentación e implementación que se podrá diagnosticar su efectividad mediante la articulación de las medidas y acciones de estos distintos componentes.

Como se ha planteado en el presente trabajo, los enfoques de emergencia y soberanía alimentaria responden a lógicas que, en la práctica, no han podido todavía ser articuladas. En la lógica lineal, las acciones directamente vinculadas a la soberanía alimentaria se interpretan como paliativos, como ayudas parciales y complementarias de la acción asistencialista que tiene el lugar central en la agenda. Sin embargo, si la apuesta a la agroecología y la agricultura familiar pueden parecer hoy como limitados aportes para garantizar el derecho a la alimentación, es porque están inescindiblemente ligadas a problemáticas más complejas que no alcanzan todavía a ingresar a la agenda política, como el acceso a la tierra, la infraestructura rural y la propiedad intelectual de las semillas. En esta lógica, el PACH no llega tampoco a abarcar otras acciones imprescindibles para una discusión profunda de la malnutrición infantil, como sería 
la regulación especial de la publicidad de alimentos destinados a esta población, considerada horizontalmente vulnerable.

Comprender la complejidad del problema del hambre y su anclaje estructural requiere desvincular su regulación del contexto de emergencia y desligarlo discursivamente de la pobreza, la vulnerabilidad y la noción de riesgo. No se desconoce que la malnutrición afecta de manera particular a los grupos sociales económicamente más vulnerables, pero en tanto problema sanitario presenta un alcance más amplio. Si la seguridad y la soberanía alimentaria constituyen los puntos de partida de la regulación de la problemática del hambre, la política alimentaria diseñada para atacarla debe afrontar también discusiones regulatorias complejas, como por ejemplo la propiedad intelectual de las semillas, el acceso y la gestión del agua o el uso de agroquímicos para la producción de alimentos.

\section{Bibliografía}

Abeyá Gilardón, E. O. (2016). Una evaluación crítica de los programas alimentarios en Argentina. Salud Colectiva, 12(4), 589-604. https://doi.org/10.18294/sc.2016.935

Aguirre, P. (2004). Ricos flacos y gordos pobres: La alimentación en crisis. Capital Intelectual.

Aguirre, P. (2005). Contribución para el diseño de una política alimentaria (El Plan Fénix en vísperas del segundo centenario: Una Estrategia Nacional de desarrollo con equidad).

Aguirre, P. (2007). Las transiciones alimentarias en el tiempo de la especie. Una mirada desde la antropología. En J. Braguinsky, Obesidad, saberes y conflictos.

Aguirre, P. (2009). La construcción social del gusto en el comensal moderno. En M. Katz, M. Bruera, \& P. Aguirre, Comer: Puentes entre la alimentación y la cultura (pp. 15-60). Libros del Zorzal.

Aulicino, C., \& Díaz Langou, G. (2012). La implementación del Plan Nacional de Seguridad

Alimentaria en ámbitos subnacionales (Documento de Trabajo $\mathrm{N}^{\circ}$ 88). CIPPEC. 
Balbín, C. F. (2015). Manual de Derecho Administrativo ( $3^{\circ}$ ). La Ley. Barruti, S. (2013). Malcomidos: Cómo la industria alimentaria argentina nos está matando. Grupo Planeta Spain.

Bojic, D., Vidar, M., Knuth, L., \& Rae, I. (2010). Guía para legislar sobre el derecho a la alimentación. Derecho a la Alimentación. Roma: FAO. Retrieved from http://www.fao.org/docrep/012/ i0815s/i0815s00.htm

Bonet de Viola, A. M. (2014). Entre seguridad, soberanía y democracia alimentaria: Consecuencias políticas de una disgregación terminológica. Derecho y Justicia, 4, 7-23.

Borrás, G., \& García, J. (2013). Políticas alimentarias en Argentina, derechos y ciudadanía. Revista Interdisciplinaria de Estudios Agrarios, 39, 111-136.

Breining-Kaufmann, C. (1991). Hunger als Rechtsproblem: Völkerrechtliche Aspekte eines Rechtes auf Nahrung. Völkerrechtliche Aspekte eines Rechtes auf Nahrung. Zürich: Schulthess TS - WorldCat.

Britos, S., O’Donnell, A., Vanina, U., \& Clacheo, R. (2009). Programas alimentarios en Argentina. CESNI Centro de Estudios Sobre Nutricion Infantil, 8-10, 8-12. https://doi.org/10.1896/020.010.0106

Britos, S., Saraví, A., \& Vilella, F. (Eds.). (2013). Alimentación saludable en la Argentina: Logros y desafíos. Orientación Gráfica Editora.

Buschini, J. (2015). La alimentación como problema en la Argentina de las décadas de 1930 y 1940. Notas para su delimitación como objeto de estudio. Cuestiones de Sociología, 12. http:// www.cuestionessociologia.fahce.unlp.edu.ar/article/view/ CSn12a08

Cardoso, J. C. (2015). Entrevista al Coordinador del Frente Parlamentario de Uruguay y

Presidente de la Comisión de Agricultura, Ganadería y Pesca del PARLATINO [Video]. https://www.youtube.com/watch?v= BQPKZYEkthE

Cartwright Traylor, J. (1988). FAO and the right to food. In A. Eide, W. B. Eide, S. Goonatilake, J. Gussow, \& Omawale (Eds.), Food as a human right (2nd ed., pp. 187-218). Tokyo; Japan: United Nations University. 
Cittadini, R. (2014). Limitaciones y potencialidades de la agroecología: Enseñanzas de una experiencia en gran escala basada en los principios de la agroecología, el ProHuerta en Argentina. En V. Hernández, F. Goulet, M. Daniele, \& N. Girard (Eds.), La agroecología en Argentina y en Francia: Miradas cruzadas (pp. 117-132). INTA.

CDESC, Comité de Derechos Económicos, S. y C. (1999). Observación general 12: Cuestiones sustantivas que se plantean en la aplicación del Pacto Internacional de Derechos Económicos, Sociales y Culturales. United Nations Economic and Social Council, pp. 1-11.

Craven, M. (1995). The International Covenant on Economic, Social and Cultural Rights: A perspective on its development. Oxford: Clarendon Press.

Fanzo, J. (2016). Policies' Food Roles on Nutrition Goals and Outcomes: Connecting of Food and Public Health Systems. En G. Steier \& K. K. Patel, International Food Law and Policy (pp. 213-251). Springer.

FAO. (1996). Informe de la Cumbre Mundial sobre la Alimentación. Roma.

Filardi, M., \& Prato, S. (2018). Reclamar el futuro de la alimentación: Cuestionando la desmaterialización de los sistemas alimentarios. En A. Morena, Cuando la alimentación se hace inmaterial: Afrontar la era digital (pp. 6-15). Red Mundial por el Derecho a la Alimentación y a la Nutrición.

Fischler, C. (2010). Gastro-nomía y gastro-anomía. Sabiduría del cuerpo y crisis biocultural de la alimentación moderna. Gazeta de Antropología, 26.

Grassi, E. (2003). El asistencialismo en el Estado Neoliberal. La experiencia Argentina de la década del 90. E-L@tina. Revista Electrónica de Estudios Latinoamericanos, 1(4), 28-51. Retrieved from https://publicaciones.sociales.uba.ar/index.php/ elatina/article/view/2712/pdf_84

Haugen, H. M. (2007). The right to food and the TRIPS Agreement: With a particular emphasis on developing countries' measures for food production and distribution. (Vol. 30). Leiden; Boston: Martinus Nijhoff Publishers. 
Maceira, D., \& Stechina, M. (2011). Intervenciones de política alimentaria en 25 años de democracia en Argentina. Revista Cubana de Salud Pública, 37(1), 44-60. https://doi.org/10.1590/ s0864-34662011000100006

Marichal, M. E. (2016). El desarrollo del Sistema Nacional de Control de Alimentos en Argentina. Revista de Derecho Agrario y Alimentario, 68(XXVIII), 157-186.

Nestle, M. (2007). Food Politics: How the Food Industry Influences Nutrition and Health, Revised and Expanded Edition. University of California Press.

O'Donnel, A., \& Britos, S. (2002). Reflexiones y propuestas en la emergencia alimentaria. Arch.Argent.Pediatr, 100(5), 412-422.

ONU/FAO. (2010). El derecho a la alimentación adecuada. Folleto Informativo No 34, p. 66. https://doi.org/1014-5613

Parlamento Latinoamericano, \& FAO. (2012). Ley Marco Derecho a la Alimentación, Seguridad y Soberanía Alimentaria. XVIII Asamblea Ordinaria del Parlamento Latinoamericano.

Pomar, A., \& Tendero, G. (2016). Respuestas transformadoras a la emergencia alimentaria. Soberania Alimentaria, 26, 39-41.

Quintana, V. M. (2008). Para recuperar nuestra soberanía alimentaria. Universidad Autónoma de Nuevo León.

Shiva, V. (2003). Cosecha Robada: El Secuestro Del Suministro Mundial de Alimentos. Editorial Paidós.

Sordini, M. V. (2014). Una revisión sobre los programas alimentarios nacionales aplicados a comedores escolares y comunitarios desde los años ochenta en Argentina. De Prácticas y Discursos. Cuadernos de Ciencias Sociales, 3(3), 1-12.

Teubal, M., \& Palmisano, T. (2013). Crisis alimentaria y crisis global: La Argentina de 2001/2002 y después. Realidad Económica, 279, 47-74.

Teubal, M., \& Rodríguez, J. (2002). Agro y Alimentos en la Globalización: Una Perspectiva Crítica. Editorial La Colmena.

Vía Campesina. (2017, octubre 26). Las administraciones gubernamentales no brindan seguridad alimentaria a las comunidades marginales. La desnutrición a causa de la pobreza va en aumento. https://viacampesina.org/es/seguridad-soberaniaalimentaria/ 